\title{
MOTIVAÇÕES DO PACIENTE RENAL PARA A ESCOLHA A DIÁLISE PERITONEAL AMBULATORIAL
} CONTÍNUA

MOTIVATIONS OF RENAL PATIENT TO CHOOSE THE CONTINUUM AMBULATORIAL PERITONEAL DIALISE MOTIVACIONES DEL PACIENTE RENAL PARA LA ELECCIÓN DE LA DIÁLISIS PERITONEAL AMBULATORIA CONTINUA

Hiarlene Gonçalves Silva ${ }^{1}$
Maria Josefina da Silva

RESUMO: A doença renal impõe ao seu portador uma nova concepção devida. Hoje, modernas técnicas, possibilitam maior liberdade ao cliente, sendo uma delas, Diálise Peritoneal Ambulatorial contínua. Objetivamos estudar a eleição pelo método e os sentimentos com relação a esta escolha pelos clientes. Concluímos que o método, apesar de suas vantagens, ainda não é o de primeira opção, os pacientes, em alguns casos não estão bem treinados com intercorrências, mas a maioria está satisfeita com a escolha. A enfermagem ainda participa pouco deste processo mas tem um papel importante no acompanhamento ambulatorial e domiciliar deste cliente, necessitando maior formação específica.

PALAVRAS CHAVES: Diálise Peritoneal; Métodos Terapêuticos; Cuidados de Enfermagem.

ABSTRACT: The renal disease imposes to its porter a new conception due. Nowadays, modern techniques, make possible more freedom to the client, being one of them, continuum Dialise peritoneal ambulatorial. Our objective is to study the election by the method and the feelings related to this chose by the client. We conclude that ther method, despite its advantages, is not the first option yet, the patient, in some cases are not well trained with intercorrences, but most of them are satisfied with the chose. The nursing still participates just a bit of this process but it has important role in the ambulatorial and domiciliar attendance of this client, needing more specific formation.

\section{KEY WORDS: Peritoneal Dialisis; Therapeutic Methods; Nursing Care}

RESUMEN: La enfermedad renal le impone a su portador una nueva concepción de vida. Hoy, algunas técnicas modernas, le posibilitan una libertad mayor al cliente, siendo una de ellas, la Diálisis Peritoneal Ambulatoria Continua. Tuvimos el objetivo de estudiar la elección de este método y los sentimientos de los clientes en relación a dicha elección. Llegamos a la conclución de que el método, a pesar de tener sus ventajas, todavía no es la primera opción, los pacientes, en algunos casos no están bien entrenados con contingencias, pero la mayoría está satisfecha con la elección. Los enfermeros todavía participan poco de este proceso pero tienen un papel importante en el acompañamiento ambulatorio y domiciliar de este cliente, necesitando una mayor formación específica.

TERMINOS CLAVES: Diálisis Peritoneal; Métodos terapéuticos; Cuidados de Enfermería.

\section{INTRODUÇÃO}

A falência renal pode afetar qualquer pessoa e pode ocorrer a qualquer momento da vida. A doença normalmente progride de forma lenta, mas gradual, resultando em múltiplos sinais e sintomas decorrentes da incapacidade do rim de manter a homeostasia interna.

A insuficiência Renal Crônica- IRC, é uma doença onde há uma perda progressiva e, geralmente, irreversível da função renal de depuração, sendo qualificada em leve, moderada e grave ou terminal. (BARROS et al, 1999). Nessas circunstâncias o rim não consegue realizar sua função filtradora, isto é, retirar do sangue todas as escórias metabólicas, como uréia e creatinina que chegam a ele, e que devem ser eliminados na urina, além de influenciar em outras importantes funções no organismo, como produção de eritropoetina e da forma ativa de vitamina D.

Embora não exista cura para a falência renal, estão disponíveis várias modalidades de tratamento:
Hemodiálise (HD): consiste na filtração do sangue através de um processo extracorpóreo de depuração mediado pela membrana de um deslizador, que funciona como um rim artificial. A duração de cada sessão desta terapêutica varia de 3 a 4 horas, 3 vezes por semana. Para a realização deste tratamento, torna-se necessário a utilização de um acesso vascular, que pode ser temporário, como os cateteres de vaso profundo de duplo lúmen, ou permanentes: fístula artério-venosa. Diálise Peritoneal Intermitente DPI: realizada 2 vezes por semana, com duração mínima de 24 horas, com tempo de permanência de 30 minutos, necessitando de ambiente hospitalar e pessoal treinado. Diálise Peritoneal Ambulatorial Contínua -DPAC: permite a realização em domicílio pelo paciente e/ou responsável, sendo 4 trocas diárias, com tempo de permanência de 4 a 6 horas. Diálise Peritoneal Contínua Assistida por Cicladora -CCPD ou Diálise Peritoneal Automática - DPA: realizada a noite durante o sono do paciente, ficando este conectado a uma máquina cicladora automática que

\footnotetext{
${ }_{1}^{1}$ Graduanda em Enfermagem pela Universidade Federal do Ceará.

${ }^{2}$ Doutora em Enfermagem. Professora Adjunta do Departamento de enfermagem da Universidade Federal do Ceará
} 
periodicamente substitui a solução de diálise por uma nova, por meio da gravidade.

Os indivíduos portadores dessa doença, para manterem-se vivos, têm que ser submetidos a um destes tratamentos. Todas estas técnicas utilizam um filtro natural, o peritôneo, membrana semipermeável, que reveste o abdome e é ricamente vascularizada, o que a torna uma área ideal para realizar a diálise.

Dentre as modalidades de tratamento para a insuficiência renal, a DPAC possibilita maior independência e liberdade ao cliente, tornando-o instrumento indispensável para o seu autocuidado e a melhora da qualidade de vida. Este estudo tem como objetivos: investigar o contexto em que o paciente toma conhecimento deste tipo de tratamento dialítico; pesquisar os critérios de escolha do cliente para a terapêutica escolhida; verificar a aceitação dos clientes pelo convívio diário com a DPAC.

\section{PROCEDIMENTOS METODOLÓGICOS}

A pesquisa consiste em um estudo exploratório descritivo com análise de dados através de freqüência simples. Seu locus foi uma clínica de hemodiálise, na cidade de Fortaleza - Ce, cujo quadro funcional conta com médicos, enfermeiras, auxiliares de enfermagem, assistente social, além do pessoal de apoio administrativo e de manutenção. Atualmente a clínica possui 25 pacientes que fazem tratamento de CAPD.

A população foi constituída de 25 pacientes, porém foi utilizada uma amostra de 21 pacientes, pois; dois não tiveram condições físicas de serem entrevistados, e dois estavam viajando por ocasião coleta de dados.

Os instrumentos para coleta de dados foram: prontuário dos pacientes onde buscamos toda o histórico de saúde do paciente: doença de base, exames laboratoriais e outros e entrevista semiestruturada. $O$ acesso aos sujeitos da pesquisa foi através de telefone quando explicamos os objetivos do trabalho e o convite para participar. Posteriormente, os que concordaram, foram convidados a comparecer à clínica para a coleta de dados.

O projeto foi aprovado pelo Conselho de Ética da Universidade Federal do Ceará, sendo dadas informações necessárias sobre o propósito do trabalho, sem a ocorrência de dados físicos ou psicológicos e assegurada anonimato e o direito de interromper sua participação em qualquer momento que fosse desejado.

\section{Caracterização dos sujeitos da pesquisa}

Observamos que $12(57,1 \%)$ estão na faixa etária de 36 a 56 anos, compondo a faixa populacional dos economicamente ativos. As limitações advindas da necessidade de diálise, impedem o desempenho profissional e o convívio social destes clientes, onerando o sistema de saúde e família. Quanto ao sexo, não há diferença significativa.

São casados $13(61,9 \%)$ dos sujeitos, e a prática do CAPD, como tratamento substitutivo, requer participação e apoio familiar para maior aceitação e adaptação à terapêutica. A maioria $(71,4 \%)$ reside em Fortaleza, facilitando o acompanhamento e evolução da doença.

Verificamos também que $10 \quad(47.5 \%)$ dos participantes são analfabetos ou estudaram até o ensino fundamental incompleto, sendo este uma importante observação para a assistência de enfermagem apropriada ao perfil da clientela. A escolaridade foi incluída no estudo, por considerarmos um fator importante para a compreensão do tratamento, capacidade de aprender os conceitos básicos da técnica do CAPD e como proceder na técnica do autocuidado.

\section{DOENÇAS ASSOCIADAS À INSUFICIÊNCIA RENAL}

No que respeita a doença de base dos clientes entrevistados, $38,0 \%$ apresenta Nefroesclerose Hipertensiva, mostrando o alto índice de hipertensos que chegam a desenvolver a Insuficiência Renal Crônica (IRC). A Nefroesclerose Diabética isolada corresponde a $14.2 \%$ da clientela. Devido à fragilidade capilar causada por essa doença a perda do acesso permanente para hemodiálise (FAV) é bastante freqüente. (GROSS et al, pg. 1996)

Hipertensão e Diabetes aparecem associadas em $66.4 \%$ da clientela entrevistada que desenvolveram a Insuficiência Renal. Segundo ANDRADE (1998) este complicador pode levar à morte ou a um dano renal lento mas progressivo.Neste momento, é válido ressaltar a importância da atenção primária desenvolvida, principalmente no programa de saúde da família (PSF), para alertar os profissionais de saúde que além do controle destas patologias atentar para sinais e sintomas precoces indicativos de falência renal.

Também é alta a percentagem de doença renal crônica relacionada a Glomerulonefrite Crônica (19\%). FIALHO et al (1988, p. 166) afirmavam que a "hipertensão Arterial ocorre desde o início ou surge durante o processo de evolução da doença". Ainda foi encontrado doença renal crônica relacionada à Síndrome Nefrótica $(9,5 \%)$ e Nefrocalcinose $(4,5 \%)$.

Comparando o tempo do diagnóstico da doença com o tempo de tratamento com o CAPD observamos que $12(57.1 \%)$, há mais de 5 anos, convivem com a falência renal e apenas $10(47.6 \%)$ clientes praticam o CAPD há mais de um ano. Desta forma, concluímos que a maioria dos clientes demora a ingressar no CAPD, e só o praticam quando há necessidade de outra modalidade de tratamento, e mesmo assim, o CAPD é uma terapêutica desconhecida pela grande maioria dos pacientes. E importante ressaltar que o tratamento com CAPD não é oneroso para a clínica e a mesma possui regras operacionais de encaminhamentos de pacientes carentes para o CAPD, ajudando-lhes financeiramente quando necessário.

\section{OPCÕES DE TRATAMENTO PELOS ENTREVISTADOS}


A hemodiálise é o tratamento inicial realizado por $20(95.2 \%)$ entrevistados. Apenas uma paciente $(4.76 \%)$ não teve experiência com outro tipo de tratamento, entrando diretamente no CAPD. Deste modo, fica mais claro perceber que a Hemodiálise é o tratamento de primeira escolha para a busca do tratamento da falência renal, sendo necessário uma divulgação maior sobre o que é o CAPD.

Segundo BRUNNER \& SUDDARTH (1999, pg. 987): "Os pacientes susceptíveis as rápidas alterações hídricas, eletrolíticas e metabólicas que ocorrem durante a hemodiálise apresentam menos problemas com uma menor velocidade da diálise peritoneal".

Há uma indicação do CAPD como terapia substitutiva para clientes com Nefroesclerose Diabética, pois é uma terapia contínua e lenta, facilitando o controle da glicemia, como afirmam Castro e Cols (1986). Desta forma, há diferentes indicações e tipos de pacientes para cada tratamento, mas a maioria dos pacientes tem condições de ser bem sucedido em qualquer modalidade terapêutica. Portanto observa-se a necessidade de divulgação dos métodos dialítico existentes para maior participação do cliente no seu tratamento.

A fonte de informação sobre o método CAPD foi, predominantemente médica $17(80.9 \%)$ e apenas 2 $(9.52 \%)$ tiveram informações desta prática com a enfermeira. Deste modo, concluímos que a Enfermagem necessita participar mais efetivamente junto aos clientes, orientando-os e esclarecendo-os constantemente. Para tanto, faz-se necessário que a enfermeira não assuma unicamente $\circ$ papel assistencial, e sim que participe da avaliação inicial do paciente e da decisão sobre a terapia dialítica, em parceria com o médico.

Por opção pessoal, 03 (14.2\%), estão realizando o CAPD por entenderem ser um tratamento menos agressivo, mais compatível com seu estilo de vida, ou por estagnação física e emocional. Quando o cliente toma esta decisão, ocorre uma avaliação médica quanto a conveniência do tratamento com o grau de insuficiência renal.

A decisão pelo método deve ser partilhada pela a família, pois o conhecimento ao tratamento está restrito a sua necessidade. É necessário que sejam expostos ao cliente e família os tipos de tratamento dialítico, suas vantagens e desvantagens, alem de suas conseqüências.

Verificamos que $12(57.1 \%)$ dos participantes executam o CAPD por indicação médica não como tratamento de primeira escolha, mas por não possuírem acesso vascular para a realização hemodiálise ou devido a cardiopatias $(4.76 \%)$ e glaucoma que representa também $4,76 \%$ da clientela. Quatro (19\%) dos clientes estão no CAPD por indicação médica relacionada a outros problemas como, reação alérgica a produtos da hemodiálise, mal estar contínuo.

Desta forma, concluímos que a grande maioria dos entrevistados não conhece o CAPD logo que ingressam na Hemodiálise, demoram na escolha de outro método tentando se adaptar, porém com o tempo este tratamento acomete outros sistemas fisiológicos, causando conseqüências cardíacas, pulmonares e outras, e aí então, pensa-se em um tratamento menos agressivo, como o CAPD, o qual poderia ter sido o tratamento de escolha para a falência renal.

\section{PROCESSO ADAPTATIVO À CAPD}

Sessenta e seis por cento da clientela (14), foram treinados e realizam a troca de bolsas diárias e, para $7(33.3 \%)$, as trocas de bolsas são realizadas por algum familiar ou outra pessoa treinada. Desta forma, observamos como se faz presente o apoio familiar junto ao paciente renal que realiza o CAPD. Todo paciente ao ingressar no CAPD, passa por um período de treinamento, realizado pela enfermeira do centro de diálise, que constitui-se de aulas teóricas e práticas, adaptadas as condições do paciente e/ou familiar em assimilar os conceitos e as rotinas dos procedimentos. De acordo com o protocolo de rotina da clínica de hemodiálise, o treinamento será sempre realizado com a presença do paciente, mesmo não sendo ele o responsável pelas trocas, só em situações excepcionais, é realizado o treinamento somente com o familiar ou responsável.

Acerca da presença de outra pessoa não treinada no local da troca, foi relatado por $9(42.8 \%)$ que outras, pessoas alheias ao tratamento, já se fizeram presente durante ou após as trocas. Estes afirmaram que a causa foi apenas curiosidade. Mesmo não sendo uma contra-indicação explícita, trata-se de um fator de risco para esses clientes, pois desconhecemos as condições em que entraram no local, que momento exato interrompeu a troca, e se tiveram alguns cuidados quanto a assepsia de mais uma pessoa que se fez presente no local das trocas, portanto o maior número de pessoa no local propicia uma maior susceptibilidade ao cliente de se distrair e cometer algum erro ou acidente, aumentando o risco de complicação.

A conseqüência desta falha no controle das trocas é evidenciada quando $13(61.9 \%)$ clientes afirmam já tirem passado por alguma complicação relacionada ao CAPD. Relacionando estas complicações mais comuns, a peritonite é a que representa o maior temor para $13(61.9 \%)$ dos sujeitos da pesquisa, relatando que tiveram uma, duas ou três vezes complicações por peritonite. A peritonite é causada pela contaminação acidental do cateter, líquido ou tubo durante as trocas das bolsas, dependendo do cuidado meticuloso do cliente com a técnica e medidas assépticas. A importância deste evento é destacada por BEVILACQUA \& GUERRA (2001, p. 34): a peritonite geralmente responsiva ao tratamento, tem importante significância devido aos custos associados ao diagnóstico, hospitalização e terapia, perda de produtividade, desnutrição, falência do método com transferência para hemodiálise, em 1 a $3 \%$ dos casos, evolução com óbito do paciente".As demais intercorrências encontradas foram: infecção no orifício de saída do cateter; infecção de túnel; complicações como refluxo gastro-esofágico e técnica asséptica incorreta. A maioria destas complicações infecciosas $(28,6 \%)$, ocorreu logo no início do 
tratamento após um ano essa incidência consideravelmente $(9,5 \%)$, evidenciando a importância do treinamento dos clientes e assim, tornando-os mais aptos de identificar dificuldades e erros. Também o enfermeiros que trabalha com este tipo de tratamento deve ser qualificado para se fazer presente no processo do tratamento, para avaliação periódica da evolução dos pacientes, e quando necessário, reciclar o treinamento de acordo com a clientela. Assim, a enfermagem teria uma assistência preventiva com maiores retornos e satisfação quanto ao tratamento, isto completaria as visitas domiciliares, ambas prevenindo as complicações e não somente quando for necessário ou indicado.

Quanto aos sentimentos com relação ao tratamento de opção, mais da metade da população 16 (76.1\%), "sentem-se bem" em praticar a CAPD, isto porque relatam ter mais liberdade, ânimo e disposição para realizar atividades, como: passear e trabalhar, ficam mais tempo em casa junto a família, a qual facilita o processo de adaptação e se distanciam da "máquina", termo utilizado para se referirem a máquina de hemodiálise, tornando-se mais independente da mesma e da clínica.

Como notamos a prática da DPAC proporciona muitos benefícios, dentre outros convívio familiar e social, independência e liberdade de deslocamento para passeios e viagens longas, possibilidade de retorno das atividades profissionais, escolares e do lar, preservação dos vasos, não necessitando da punção de 6 agulhas semanais para a diálise, maior liberdade de dieta, frutas e líquidos, maior flexibilidade de horários para realizar a diálise, o paciente é treinado e orientado para o seu autocuidado, preserva a função residual por mais tempo, promovendo uma maior qualidade de vida e possibilita maior estabilidade cardíaca, menor variação do peso e maior controle da pressão arterial e anemia. Esta satisfação é comprovada pela literatura (BRUNNER \& SUDDARTH, 1999).

Se por um lado temos a satisfação do cliente, por outro temos o cansaço do cuidador. O idoso submetido a este tratamento sofre por se tornar dependente do cuidador, causando sobrecarga emocional e psico-social, além de queixas pelo isolamento dos amigos adquiridos nas sessões de hemodiálise. É importante criar um grupo de pacientes submetidos a DPAC, buscando satisfazer as necessidades de seus membros, oferecendo aceitação, apoio e auxílio mútuo, superando padrões de comportamentos mal-adaptativos, onde eles próprios busquem se ajudar e ajudar os outros.

O grande problema da DPAC, segundo os entrevistados, é a adaptação deste e da família a este tipo de tratamento domiciliar. A adaptação se deu por uma questão de sobrevivência. Pacientes relataram que ou faziam o CAPD ou ficariam em casa aguardando o dia da morte. Destes $9.5 \%$ realizam o tratamento, porém com medo de sofrer algum processo infeccioso. Neste caso a capacitação e autoconfiança devem ser fortalecidas para evitar que este sofra alguma complicação pela falta de capacitação. Outro aspecto são as intercorrências sistêmicas, conforme relato de um paciente que referiu passar mal com bolsas de diálise mais concentrada, estando o intestino mais sensível. Neste caso, é necessário uma avaliação precisa da enfermeira quanto a técnica correta durante a troca, se a concentração está correta, e iniciar um plano assistencial, juntamente com o médico, que melhore a sintomatologia deste cliente , para a aceitação do método utilizado.

A ansiedade vivenciada pelos pacientes que praticam a DPAC está relacionada a eventuais transtornos técnicos ou acidentais, que venham causar alguma infecção, porém tal ansiedade é reduzida por um treinamento e acompanhamento satisfatório, que faz com que o paciente se torne firmemente convencido da sua própria capacidade e autoconfiança em se autocuidar.

Para o paciente renal crônico que realiza o CAPD a distorção da imagem corporal causa forte impacto psicológico quanto ao seu aspecto físico, podendo prejudicar o tratamento enquanto não houver aceitação de suas conseqüências, como o aumento do abdômen com sensação de obesidade. A rejeição do cateter torna estes pacientes mais susceptíveis a infecção. BRUNNER \& SUDDARTH (1999, p. 997) a respeito disso, esclarecem que a cintura aumenta de três a cinco centímetros (ou mais) com o líquido infundido no abdômen, e isso complica para o paciente na hora de escolher uma roupa favorecendo a sensação de obesidade. A imagem corporal pode ser tão afetada que o paciente pode passar dias ou semanas sem olhar para o cateter, nem mesmo cuidar de sua limpeza.

Verificamos com a pesquisa que 17 (30.9\%) não referem dificuldades na prática e condução do tratamento dialítico e apenas 4 (19\%) relatam dificuldades econômicas, cansaço físico para realização das trocas. Neste caso, outras pessoas próximas podem ser treinadas para aliviar este cansaço fixando os horários de acordo com o seu estilo de vida.

Quanto ao nível se satisfação do tratamento, observamos também que 17 (80.9\%), não tem interesse de mudar de modalidade de tratamento e dizem:

"Só sairia deste tratamento se tivesse um rim de plástico que resolvesse o problema".

"Gostaria de ficar normal, mas como não é possível quero ficar no CAPD”.

"Este tratamento é a minha tranqüilidade, espero ficar muito tempo e jamais voltar para a hemodiálise e se isso acontecer que "o Chefe' (Deus) me leve antes."

Verificamos que 4 (19\%) da clientela, tem interesse de mudar de modalidade de tratamento, um destes clientes continua o tratamento até conseguir um transplante e os outros três, que representa $14.2 \%$ da população tem interesse pela DPA - Diálise Peritoneal Automática.

As diferenças da DPA para DPAC são: a DPA utiliza bolsas plásticas com volumes maiores (6L); 
dializa por toda à noite; a máquina controla automaticamente os ciclos de infusão, permanência e drenagem da solução, bem como memoriza os registros de volumes de sua drenagem; a infusão e drenagem são automáticas e independe da gravidade; redução do número de trocas ao dia, menor risco de infecção e ciclos de diálise mais precisos, controlados automaticamente.

Desta forma, é importante que a enfermeira avalie periodicamente o nível de adaptação dos clientes que realizam CAPD e os informe sobre outros tipos de tratamentos, vantagens e desvantagens e quais as probabilidades de mudança de tratamento.

$\mathrm{Na}$ opinião de CASAGRANDE \& CESARINO (1998), a enfermeira necessita ter, além da fundamentação científica e da competência técnica, conhecimentos dos aspectos que levam em consideração os sentimentos e as necessidades dos pacientes.Assim, o papel da enfermeira é destacado como de grande colaboração na tentativa de ajudar o paciente renal crônico a adaptar-se ao novo estilo de vida.

Por fim, o papel da equipe multiprofissional, como um todo, é buscar o mais alto bem-estar para o paciente, dentro de suas possibilidades, respeitando sua individualidade e opções, buscando assisti-lo como um todo, holisticamente, dentro do complexo biopsicosocial, tendo em mente que, em DPAC, o principal responsável pelo sucesso do tratamento será o próprio paciente.

\section{CONCLUSÕES}

A falência renal impõe uma nova rotina ao seu portador, novos hábitos e novo estilo de vida. $\mathrm{Na}$ clientela estudada, notou-se que a grande maioria dos clientes teve como causa básica da Insuficiência Renal, problemas como Hipertensão (38\%) e Diabetes $(14,2 \%)$, além da associação destas patologias. Desta forma, a assistência primária possui uma importância relevante na detecção e acompanhamento de pacientes portadores desta patologia para um alerta à pacientes com sinais e sintomas precoces indicativos de falência renal.

A CAPD um tratamento com início protelado, pois a hemodiálise, nos dados analisados, constitui o tratamento de primeira escolha, ficando esta opção terapêutica decorrente do desgaste físico, principalmente por ausência de acesso vascular. O conhecimento sobre o método veio através do médico e apenas uma minoria obteve informação da enfermeira, demonstrando a necessidade da divulgação dos tipos de métodos dialíticos, suas vantagens, desvantagens e conseqüências. $A(0)$ enfermeira(o) deve participar da avaliação inicial do paciente e da decisão sobre a terapia dialítica, junto ao médico.

A atuação da(o) enfermeira(o) como educadora e facilitadora da adaptação do cliente é indispensável, pois a mesma é responsável pelo treinamento e conscientização do cliente sobre o autocuidado, conduzindo informações a todos os clientes, tornando- os membros ativos no processo saúde-doença, sendo o paciente o responsável pelo sucesso do tratamento.

O apoio familiar é indiscutivelmente de grande importância para o cliente renal crônico em programa de CAPD, facilitando o processo adaptativo

\section{REFERÊNCIAS BIBLIOGRÁFICAS}

ANDRADE, M. M. de. Introdução a Metodologia do Trabalho Científico: elaboração do trabalho na graduação. São Paulo: Atlas 1998.

BARROS, E: MAN R. C; THOMÉ, F. S. GONÇALVES, L. F. S. Nefrologia: Rotinas, Diagnósticos e Tratamentos. 2 ed. Porto Alegre. ARTMED. 1999.

BEVILACQUA, J. L; GUERRA, E. M. M. Protocolo para DPAC. $2^{\mathrm{a}}$ Ed. São Paulo. 2001, 100pgs.

BRUNNER, L. S. \& SUDDARTH, D. S. Tratado de Enfermagem Médico - Cirúrgico._ 8 ed. Rio de Janeiro; Guanabara Koogan, 1999.

CASAGRANDE, L. D. R.; CESARINO, C. B. Paciente com Insuficiência Renal Crônica em Tratamento Hemodialítico. Atividade Educativa do Enfermeiro. Revista Latino Americana de Enfermagem. Ribeirão Preto. V. 6, no 4, p.31-40, out. 1998

FIALHO, F.; OLIVEIRA, C. A. B $\square$ L.S. RIOGA, L. Doenças dos rins- Estudo Clínico. Tratamento. In. NOGUEIRA JÚNIOR, A.; SANTOS, O. R. et. al. (Coloque o título do livro e destaque em itálico). São Paulo : Fundo editorial Byk. , 1988. Capítulo 7, p. 166 (páginas do capítulo)

Texto original recebido em: $18 / 02 / 2003$ Publicação aprovada em: 25/04/2003 